\title{
Longitudinal age-related changes in cobalamin and folate status in community-dwelling older adults
}

\author{
Alexandra Jungert ${ }^{1}$, Carola Zenke-Philippi ${ }^{2}$ and Monika Neuhäuser-Berthold ${ }^{1}$ \\ ${ }^{1}$ Justus Liebig University, Institute of Nutritional Science, Human Nutrition, Giessen, Germany and \\ ${ }^{2} J u s t u s$ Liebig University, Institute of Agronomy and Plant Breeding II, Biometry and Population Genetics, Giessen, \\ Germany
}

\section{Abstract}

Advancing age is regarded as a risk factor for an insufficient cobalamin and folate status. However, longitudinal data are lacking and little is known on the impact of age in subjects $\geq 60$ years after considering potential confounding factors. Therefore, the present study investigates age-related changes in cobalamin and folate status in older adults by using longitudinal data of 332 German subjects aged $\geq 60$ years, who participated in the longitudinal study on nutrition and health status of senior citizens in Giessen (GISELA study). All subjects had complete data records on at least three follow-ups between 1997 and 2014. The mean follow-up time was 12 years. Fasting serum concentrations of cobalamin and folate were determined with SimulTRAC-SNB radio assay kit. In each follow-up, body composition (bioelectrical impedance analysis), dietary cobalamin and folate intakes (3-day estimated dietary record), supplement use and lifestyle factors (questionnaires) were assessed. Linear mixed models were used to analyze age-related changes in serum concentrations of cobalamin and folate by considering sex, absolute fat-free mass, supplement use, dietary intakes and smoking behavior as potential confounding variables. Furthermore, due to the metabolic interaction of cobalamin and folate, linear mixed models implemented a mutual adjustment of serum cobalamin and serum folate. At baseline, $11.4 \%$ and $7.8 \%$ of the subjects had cobalamin concentrations $<148 \mathrm{pmol} / \mathrm{L}$ and folate concentrations $<10 \mathrm{nmol} / \mathrm{L}$, respectively. In contrast, dietary cobalamin intakes $<4 \mu \mathrm{g} / \mathrm{d}$ and folate intakes $<300 \mu \mathrm{g} / \mathrm{d}$ were found in $24.4 \%$ and $76.5 \%$ of the subjects, respectively. Without adjustments, a positive influence of age on serum cobalamin [parameter estimate $(95 \% \mathrm{CI})=4.57(1.67,7.46)$ ] and serum folate $[0.29(0.18,0.40)]$ was found. After multiple adjustments, age was still a positive predictor of serum folate $[0.25(0.14,0.36)]$, whereas no significant influence on serum cobalamin was found $[2.73(-0.23,5.69)]$. Similar results were noticed when the analyses were restricted to non-users of B-vitamin/multi-vitamin supplements. In conclusion, the present study in community-dwelling subjects does not confirm age-related declines in serum concentrations of cobalamin and folate between the ages of 60 and 90 years. Although a significant proportion of the subjects showed dietary intake levels below the current European references values, serum concentrations of cobalamin and folate were predominantly in reference ranges. Longitudinal investigations on other biomarkers of cobalamin and folate status are warranted.

\section{Conflict of Interest}

There is no conflict of interest. 